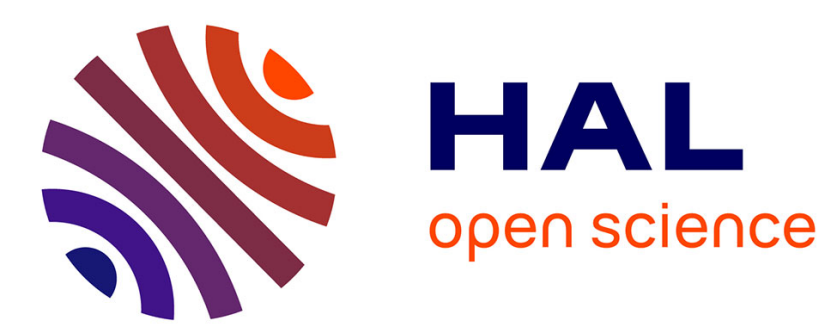

\title{
Generation of Multimodal Behaviors in the Greta platform
}

Michele Grimaldi, Catherine Pelachaud

\section{To cite this version:}

Michele Grimaldi, Catherine Pelachaud. Generation of Multimodal Behaviors in the Greta platform. 21st ACM International Conference on Intelligent Virtual Agents, Sep 2021, Kyoto (virtual), Japan. 10.1145/3472306.3478368 . hal-03428896

\section{HAL Id: hal-03428896 https://hal.science/hal-03428896}

Submitted on 15 Nov 2021

HAL is a multi-disciplinary open access archive for the deposit and dissemination of scientific research documents, whether they are published or not. The documents may come from teaching and research institutions in France or abroad, or from public or private research centers.
L'archive ouverte pluridisciplinaire HAL, est destinée au dépôt et à la diffusion de documents scientifiques de niveau recherche, publiés ou non, émanant des établissements d'enseignement et de recherche français ou étrangers, des laboratoires publics ou privés. 


\section{Generation of Multimodal Behaviors in the Greta platform}

\author{
Michele Grimaldi \\ ISIR, Sorbonne University \\ Paris, France \\ michele.grimaldi@isir.upmc.fr
}

\section{KEYWORDS}

virtual agent, FML, image schema, ideational unit, multimodal behavior

\section{INTRODUCTION}

One of the main challenges when developing Embodied Conversational Agents ECAs is to give them the ability to autonomously produce meaningful and coordinated verbal and nonverbal behaviors. Those behaviors concern facial expressions, gestures, head movement, posture, etc. These multimodal behaviors may have different communicative functions; they can stress a word, expression an emotion, indicate a point in space, to name a few functions. They are aligned with speech, showing a high degree of synchronization. There exist many computational models that can compute multimodal behaviors for virtual agent $[2,4,6,9,12,14]$. Our goal is to merge some to have a large range of behaviors to create expressive ECAs.

\section{GRETA PLATFORM}

Greta [13] is a real-time three dimensional embodied conversational agent with a 3D model of a human-like appearance compliant with MPEG-4 animation standard. It is able to communicate using a rich palette of verbal and nonverbal behaviors. Greta can talk and simultaneously show facial expressions, gestures, gaze, and head movements. It relies on procedural animation.

Two standard XML languages FML [7] and BML [8] are used to encode, respectively, its communicative intentions and behaviors based on the standard SAIBA architecture. Our current goal is, given communicative intentions specified in an FML file, to generate multimodal behaviors relying on three different computational models.

In our earlier model, Greta standard treatment instantiates the communicative intentions defined in an FML file into multimodal behaviors. The instantiation is based on a lexicon that contains pairs of the type (intention, multimodal behaviors). The instantiated behaviors are then combined and synchronized with speech; they are sent to the Behavior Realizer module that produces the final animation of the virtual agent.

That process is at the basis of all Greta's behaviors but is limited in terms of behaviors complexity.

We present now the two other modules we have added. We first introduce the concept of Ideational Unit.

2021-07-02 15:14. Page 1 of 1-3.

\author{
Catherine Pelachaud \\ CNRS, ISIR, Sorbonne University \\ Paris, France \\ catherine.pelachaud@upmc.fr
}

\section{IDEATIONAL UNIT}

Ideational Units are units of meaning that give rhythm to the discourse of a person and during which gestures show similar properties [16].

A gesture shape can have invariant shape properties that are critical for its meaning [16]. For instance, a gesture representing an ascension would probably have an upward movement but the hand shape may not be of particular relevance (for conveying the ascension meaning).

Within an Ideational Unit, successive gestures need to show significant changes to be distinguished. However, invariant properties of a gesture can be transferred to the variant properties of the surrounding gestures [16]. As such within an Ideational Unit, successive gestures may share common conformational features while still being distinguishable by other features.

\section{MEANING MINER MODULE}

Meaning Miner module [15] treatment is based on the concepts of Images Schemas[1] and Ideational Unit [3] as the intermediate language between the verbal and nonverbal channels.

The Meaning Miner module takes as input the FML file marked with prosodic and Ideational Unit [3]. Then it finds the corresponding image schemas and builds the corresponding gesture [??]. The Image Schemas Extraction module has the task of identifying the Image Schemas from the surface text of the agent's speech and to align them with the spoken utterance (for future gesture alignment).

After obtaining a list of aligned Image Schemas for a sequence of spoken text, the gesture modeler module builds the corresponding gestures. The first step is to retrieve the gesture invariants to build the final gestures. Gestures are described by several features, namely hand shape, wrist orientation, movement and position in gesture space [10]. As explained in Section 3, gesture invariants are conformational features of gesture (e.g. a hand shape) that need not to be altered to express a given meaning and that remains invariant in successive gestures within a same temporal unit [16].

For each Image Schema we search which features are needed to express its meaning and how it is expressed using a dictionary that maps each Image Schema into its corresponding invariants.

The Meaning Miner module has to co-articulate gestures within an Ideational Unit, that structures speech, by computing either a hold or an intermediate relaxed pose between successive gestures (instead of returning to a rest pose); it also has to transfer properties of the main gesture onto the variant properties of the other gestures of the same Ideational Unit. Doing so allows us to ensure that a 
meaning expressed through an invariant is carried on the same hand throughout an Ideational Unit [17].

\section{$5 \quad$ NVBG}

To extend the range of behaviors that Greta's agent can assume, the NVBG (Non Verbal Behavior Generator) [11] module has been integrated in the Greta platform. Greta launches $N V B G$ module and its charniak parser [5]. It sends the text to be said to the $N V B G$ module as a vrExpress Message. In the $N V B G$ module, gestures are distinguished by:

- animation: that concerns the whole body movement except for the head

- head: that involves head movement such as nod or shake

In NVBG, an animation is a macro-type that conveys communicative acts such as: contemplate, negation, contrast, response, request, listening and so on. If $N V B G$ finds an animation or head movement that is linked a such a communicative act, it will send back the encrypted response of the treatment.

The animation tag, the macro-type and the name of the gesture that NVBG outputs are directly not recognized in the Greta platform. The animations computed with the $N V B G$ module are motion capture data. For that reason the response of the $N V B G$ module is treated to suit the XML elements and gesture names understandable by Greta. Two mapping files are used to map the name of $N V B G$ 's gesture and types to Greta's gesture and types.

Each animation outputted by the NVBG module is an XML line that has some attributes. Firstly the mapping files allow us to change the $N V B G$ gesture type and name with the corresponding gesture in Greta. Then the attributes that are not useful for Greta are removed or modified.

The NVBG module defines only the start of a gesture at a certain time marker without defining when it should finish (as it is defined by the corresponding motion capture animation file). Thus, when translating the output of the NVBG module to Greta, as Greta uses procedural animation, an end attribute is added for each animation line. The full treated response is a series of XML lines with tags linked to APML-FML entries (eg deictic, iconic, performative) that are understandable by Greta [16].

Now the Behavior Planner module can understand the gestures computed from NVBG. It then needs to translate them into BML signals. The whole process can be summarized as a transformation of mocap animation into BML signals that are defined by phases (such as stroke-start and stroke-end phases [8]. Finally theBehavior Realizer module receives all the signals and computes the animation via the MPEG-4 player.

\section{INTEGRATION}

The integration that has been done allows Greta to use Meaning Miner and NVBG models independently and to combine the results of their treatments with the standard treatment that is done by default. The two modules are fully integrated within the Greta platform.

\subsection{Architecture}

The Greta architecture has been updated to allow it to access the treatment of both modules, NVBG and Meaning Miner. The two models are added as external modules. The FML module reads an FML file and interacts sequentially with the three behavior generation modules. The gestures that are computed by each of them are sent to the Behavior Planner modules that orders and synchronizes them.

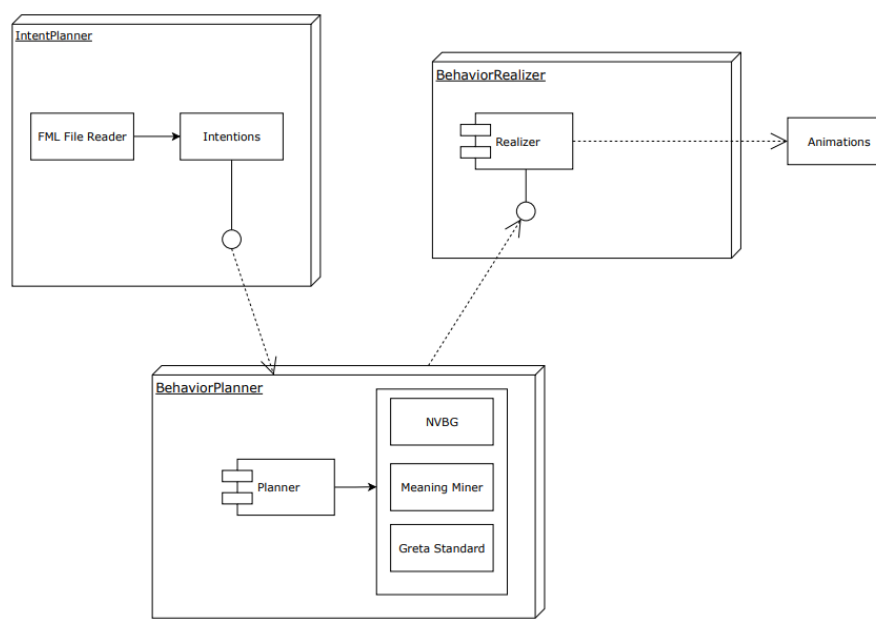

Figure 1: Updated treatment within the Behavior Planner module

The Behavior Planner module is re-designed to contain the NVBG and the Meaning Miner models. The remaining of the animation computation process, involving translation of the gesture into signals and then signals into video-audio animation, is not affected. The Behavior Planner module now contains all the treatments. Even if one of them does not find gestures this does not affect the rest of the process.

\section{CONCLUSION AND FUTURE WORKS}

The redesigned architecture integrates three modules to compute multimodal behaviors to convey communicative intentions. It allows a virtual agent to display a larger spectrum of behaviors. In the foreseeable future we intend to add another model to the agent architecture relying on a machine learning approach, namely the Sequence-to-Sequence Predictive Model [18]. It will allow Greta to extend its range of gesture and improve gestural performances for life-like virtual characters. Examples of animation can be found in the demo video at:

https://user-images.githubusercontent.com/49474878/11729617008fae880-ae75-11eb-941d-d36012a870fc.mp4

So far, we are using in-house procedural animation. We are currently integrating the Greta platform within Unity3D to be able to use the animation capabilities of the game engine. 


\section{ACKNOWLEDGMENT}

We thank Philippe Gauthier for his help and support. We are very greatful to Stacy Marsella and Jinah Lee for giving us access to the code of $N V B G$

\section{REFERENCES}

[1] C. Clavel B. Ravenet, C. Pelachaud. 2018. Automatic Nonverbal Behavior Generation from Image Schemas. 9 (July 2018).

[2] Kirsten Bergmann and Stefan Kopp. 2009. GNetIc-Using bayesian decision networks for iconic gesture generation. In International Workshop on Intelligent Virtual Agents. Springer, 76-89.

[3] Geneviève Calbris. 2011. Elements of meaning in gesture. Vol. 5. John Benjamins Publishing.

[4] J. Cassell, H. Vilhjálmsson, and T. Bickmore. 2001. BEAT: the Behavior Expression Animation Toolkit. In Computer Graphics Proceedings, Annual Conference Series. ACM SIGGRAPH

[5] Eugene Charniak. 2002. A Maximum-Entropy-Inspired Parser. Proc NAACL 1 (05 2002)

[6] Ylva Ferstl, Michael Neff, and Rachel McDonnell. 2019. Multi-objective adversarial gesture generation. In Motion, Interaction and Games. 1-10.

[7] Dirk Heylen, Stefan Kopp, Stacy Marsella, Catherine Pelachaud, and Hannes Vilhjálmsson. 2008. The Next Step towards a Function Markup Language. International Workshop on Intelligent Virtual Agents, 270-280. https://doi.org/10. 1007/978-3-540-85483-8_28

[8] Stefan Kopp, Brigitte Krenn, Stacy Marsella, Andrew Marshall, Catherine Pelachaud, Hannes Pirker, Kristinn Thórisson, and Hannes Vilhjálmsson. 2006. Towards a Common Framework for Multimodal Generation: The Behavior Markup Language. Intelligent Virtual Agents, Springer LNCS 4133, 205-217. https://doi.org/10.1007/11821830_17
[9] Taras Kucherenko, Patrik Jonell, Sanne van Waveren, Gustav Eje Henter, Simon Alexandersson, Iolanda Leite, and Hedvig Kjellström. 2020. Gesticulator: A framework for semantically-aware speech-driven gesture generation. In Proceedings of the 2020 ICMI.

[10] Silva Ladewig and Jana Bressem. 2013. Linguistic perspective on the notation of gesture phases. 1060-1079.

[11] Jina Lee and Stacy Marsella. 2006. Nonverbal Behavior Generator for Embodied Conversational Agents. 243-255. https://doi.org/10.1007/11821830_20

[12] Stacy Marsella, Yuyu Xu, Margaux Lhommet, Andrew Feng, Stefan Scherer, and Ari Shapiro. 2013. Virtual character performance from speech. In Proceedings of the 12th ACM SIGGRAPH/Eurographics Symposium on Computer Animation. 25-35.

[13] Isabella Poggi, Catherine Pelachaud, F. Rosis, Valeria Carofiglio, and Berardina Carolis. 2005. Greta. A Believable Embodied Conversational Agent. 3-25. https: //doi.org/10.1007/1-4020-3051-7_1

[14] Brian Ravenet, Chloé Clavel, and Catherine Pelachaud. 2018. Automatic nonverbal behavior generation from image schemas. In Proceedings of the 17th internationa conference on autonomous agents and multiagent systems. 1667-1674.

[15] Brian Ravenet, Catherine Pelachaud, Chloé Clavel, and Stacy Marsella. 2018. Automating the Production of Communicative Gestures in Embodied Characters. FRONTIERS IN PSYCHOLOGY (2018)

[16] Jürgen Streeck. 2013. Elements of Meaning in Gesture, Geneviève Calbris John Benjamins Publishing Company (2011), pp. 378 + VIII. Price: EUR 95.00 | USD 143.00, ISBN: 978-90-272-2847-5. Lingua 134 (09 2013). https://doi.org/10.1016/j. lingua.2013.06.005

[17] Yuyu Xu, Catherine Pelachaud, and Stacy Marsella. 2014. Compound gesture generation: A model based on ideational units. In International Conference on Intelligent Virtual Agents. Springer, Cham, 477-491.

[18] Fajrian Yunus, Chloé Clavel, and Catherine Pelachaud. 2020. Sequence-toSequence Predictive Model: From Prosody To Communicative Gestures. (08 2020) 